\title{
Association of Serum Potassium with All-Cause Mortality in Patients with and without Heart Failure, Chronic Kidney Disease, and/or Diabetes
}

\author{
Allan J. Collins ${ }^{a} \quad$ Bertram Pitt $^{b} \quad$ Nancy Reaven ${ }^{c}$ Susan Funk \\ Karen McGaughey ${ }^{d}$ Daniel Wilson ${ }^{e}$ David A. Bushinsky ${ }^{f}$ \\ ${ }^{a}$ Nephrology Research, University of Minnesota, Minneapolis, MN, ${ }^{b}$ Internal Medicine, University of Michigan, Ann \\ Arbor, MI, 'Strategic Health Resources, La Canada, CA, dCalifornia Polytechnic State University, San Luis Obispo, \\ and e Relypsa, Inc., a Vifor Pharma Group Company, Redwood City, CA, and ${ }^{\mathrm{f}}$ Nephrology Research, University of \\ Rochester, Rochester, NY, USA
}

\section{Keywords}

Hyperkalemia · Hypokalemia · Chronic kidney disease . Heart failure $\cdot$ Diabetes $\cdot$ Mortality

\begin{abstract}
Background: The relationship between serum potassium, mortality, and conditions commonly associated with dyskalemias, such as heart failure (HF), chronic kidney disease (CKD), and/or diabetes mellitus (DM) is largely unknown. Methods: We reviewed electronic medical record data from a geographically diverse population $(n=911,698)$ receiving medical care, determined the distribution of serum potassium, and the relationship between an index potassium value and mortality over an 18-month period in those with and without HF, CKD, and/or DM. We examined the association between all-cause mortality and potassium using a cubic spline regression analysis in the total population, a control group, and in HF, CKD, DM, and a combined cohort. Results: $27.6 \%$ had a potassium $<4.0 \mathrm{mEq} / \mathrm{L}$, and $5.7 \%$ had a value $\geq 5.0 \mathrm{mEq} / \mathrm{L}$. A U-shaped association was noted between serum potassium and mortality in all groups, with lowest allcause mortality in controls with potassium values between 4.0 and $<5.0 \mathrm{mEq} / \mathrm{L}$. All-cause mortality rates per index potassium between 2.5 and $8.0 \mathrm{mEq} / \mathrm{L}$ were consistently greater with HF $22 \%$, CKD $16.6 \%$, and DM $6.6 \%$ vs. controls $1.2 \%$,
\end{abstract}

and highest in the combined cohort $29.7 \%$. Higher mortality rates were noted in those aged $\geq 65$ vs. $50-64$ years. In an adjusted model, all-cause mortality was significantly elevated for every $0.1 \mathrm{mEq} / \mathrm{L}$ change in potassium $<4.0 \mathrm{mEq} / \mathrm{L}$ and $\geq 5.0 \mathrm{mEq} / \mathrm{L}$. Diuretics and renin-angiotensin-aldosterone system inhibitors were related to hypokalemia and hyperkalemia respectively. Conclusion: Mortality risk progressively increased with dyskalemia and was differentially greater in those with HF, CKD, or DM.

(C) 2017 The Author(s) Published by S. Karger AG, Basel

\section{Introduction}

Potassium is the most abundant intracellular cation and it plays a key role in the cellular function of nerve and muscle tissue. Potassium disorders or dyskalemias are relatively common in clinical practice. Hypoand hyperkalemia are important electrolyte abnormalities, as both may contribute to the development of serious or life-threatening cardiac arrhythmias and death, especially in patients with cardiovascular or renal disease.

Long-term maintenance of potassium homeostasis is largely achieved by renal excretion of potassium in re-

\begin{tabular}{ll}
\hline KARGER & $\begin{array}{l}\text { ( ) 2017 The Author(s) } \\
\text { Published by S. Karger AG, Basel }\end{array}$ \\
$\begin{array}{l}\text { E-Mail karger@karger.com } \\
\text { www.karger.com/ajn }\end{array}$ & $\begin{array}{l}\text { This article is licensed under the Creative Commons Attribution- } \\
\text { NonCommercial-NoDerivatives 4.0 International License (CC BY- } \\
\text { NC-ND) (http://www.karger.com/Services/OpenAccessLicense) } \\
\text { Usage and distribution for commercial purposes as well as any dis- }\end{array}$
\end{tabular}


sponse to variations in dietary intake [1]. Disease states such as heart failure (HF), chronic kidney disease (CKD), and diabetes mellitus (DM), and drug therapy for these conditions, may interfere with the kidney's ability to match potassium intake with potassium excretion and contribute to dyskalemias.

Hypokalemia generally occurs with diuretic therapy for hypertension (HTN) or HF. Unrecognized hypokalemia is a leading cause for iatrogenic morbidity and mortality in cardiac patients who have an inherent risk for arrhythmia and who are prescribed medications that increase risk for hypokalemia [2]. Potassium concentrations $<3.5 \mathrm{mEq} / \mathrm{L}$ have been associated with a higher risk of atrial fibrillation, while Q-T interval prolongation, risk of torsade des pointes, ventricular fibrillation, and sudden cardiac death may occur with potassium values $<3.0 \mathrm{mEq} / \mathrm{L}[3,4]$. Although hypokalemia has generally been defined on the basis of serum potassium concentrations $<3.5 \mathrm{mEq} / \mathrm{L}$, evidence suggests that mild reductions in serum potassium $(3.5-<4.0 \mathrm{mEq} / \mathrm{L})$ may be associated with excess mortality in patients with $\mathrm{HF}$ and/or CKD [5-7].

Hyperkalemia generally develops with impaired renal function, and individuals with HF or DM and advanced $\mathrm{CKD}$ are at greatest risk [8]. Potassium levels $>6.0 \mathrm{mEq} / \mathrm{L}$ have been associated with peaked $\mathrm{T}$ waves, wide QRS complexes, bradycardia, asystole, and sudden death [4]. Drug therapy with renin-angiotensin-aldosterone inhibitors (RAASi) has been implicated as a major cause of hyperkalemia, especially in patients with cardiac or renal disease.

We analyzed an electronic medical record (EMR) database and determined the distribution of serum potassium, and prevalence of $\mathrm{HF}, \mathrm{CKD}, \mathrm{DM}$, cardiovascular disease (CVD), acute myocardial infarction (AMI), HTN, and prescriptions for medications of interest in a large, geographically diverse US population receiving medical care. We then determined the association between an index potassium value and all-cause mortality in the study sample, in cohorts with HF, CKD, DM and all 3 conditions compared to a control group, and in a population $\geq 65$ years.

\section{Methods}

\section{Data Sources}

We utilized de-identified EMR data on approximately 7 million patients collected from multiple US integrated health delivery networks (Humedica, Boston, MA, USA). Humedica aggregates longitudinal outpatient and hospital data from medical care systems located across the United States into a comprehensive clinical database, and includes data on those covered by private insurance, Medicare, and Medicaid, and on the uninsured. Our review identified $1,716,141$ individuals $\geq 5$ years of age with at least 2 serum potassium measurements from 2007 to 2012 (online suppl. Fig. S1; for all online suppl. material, see www.karger. com/doi/10.1159/000479802). Demographic data, medical diagnoses, procedural data, selected laboratory studies, and outpatient prescriptions were collected from outpatient, hospital, and emergency department visits. Patient age was calculated from birth year; those born before 1924 were assigned 1924 due to database restrictions. Deaths were identified from the United States Social Security Death Index prior to the de-identification of the data set, and were augmented by deaths identified from hospital and EMR elements [9]. Information on date of death, race, ethnicity, and the EMR of patients with $<2$ potassium measurements were censored by Humedica.

\section{Serum Potassium}

A single serum potassium measurement, the last potassium value recorded prior to the study entry date of January 1, 2012, was utilized as the index or baseline value for all outcome analyses. The date of this baseline potassium value was used for determining age and as a cutoff for collection of pre-study medical history. Potassium measurements $<2.5$ or $>10 \mathrm{mEq} / \mathrm{L}$ were infrequent and were excluded from analysis secondary to the potential for laboratory error. Baseline potassium values were generally obtained during outpatient visits. However, $11 \%$ were obtained in emergency departments and $9 \%$ during hospitalization. Serum potassium was measured in multiple laboratories.

\section{Inclusion and Exclusion Criteria and Data Collection}

Individuals with incomplete demographic data and/or less than 2 years of pre-baseline medical records were excluded, as were those with end-stage renal disease and acute kidney injury; conditions frequently accompanied by wide fluctuations in potassium. A total of 759,029 subjects were excluded for insufficient data, and 45,414 for the above diagnoses. The study sample comprised of 911,698 individuals who had a baseline potassium value, complete demographic data, and a pre-study medical record.

We utilized predefined ICD-9 diagnostic codes to identify $\mathrm{HF}, \mathrm{CKD}$, and DM, as well as CVD diagnoses of AMI, ischemic heart disease, stroke, peripheral vascular disease, other atherosclerosis, and HTN (online suppl. Table S6). Laboratory, medical procedures and prescription history were used to supplement ICD-9 diagnoses (LVEF, MDRD eGFR, glycosylated hemoglobin, and hypoglycemic medications). Disease state classification was based on one or more recorded diagnoses or events over a 2 -year period prior to baseline. Outpatient prescriptions for RAASi, defined as any written prescription for an angiotensinconverting enzyme or angiotensin receptor blocker, mineralocorticoid antagonist, or direct renin inhibitor - and for all diuretics were captured, without respect to dose, over a 12-month period prior to baseline potassium. We identified 4 non-exclusive disease state cohorts of interest: HF, CKD (stage 3-5), DM, and with all 3 conditions.

Our control group comprised of individuals without known HF, CKD, DM, CVD, or HTN. Two age-specific cohorts, age between 50 and 64 and $\geq 65$ years, were examined to determine the association of age on the potassium-mortality relationship. 
Statistical Analyses

Baseline demographic, disease state, and prescribed medication data were reported per each $0.5 \mathrm{mEq}$ increase in serum potassium from 2.5 to $8.0 \mathrm{mEq} / \mathrm{L}$, for the study sample. We performed univariate and multivariate analyses on serum potassium values from 2.5 to $8.0 \mathrm{mEq} / \mathrm{L}$ for each $0.1 \mathrm{mEq}$ change, to examine the association between baseline potassium and age, gender, CVD, AMI, HTN, HF, CKD, DM, RAASi, and diuretics. Due to a U-shaped relationship between serum potassium and mortality, predictors of hypo- or hyperkalemia were evaluated separately. Multivariate regression analyses generated fully adjusted odds ratios for serum potassium values $>5.0 \mathrm{mEq} / \mathrm{L}$ among subjects with baseline potassium concentrations $\geq 4.0 \mathrm{mEq} / \mathrm{L}$, and fully adjusted ratios for serum potassium $<4.0 \mathrm{mEq} / \mathrm{L}$ and $<3.5 \mathrm{mEq} / \mathrm{L}$ among subjects with baseline potassium concentrations $\leq 5.0 \mathrm{mEq} / \mathrm{L}$.

\section{Death Event and Modeling}

The primary outcome was all-cause death, recorded following baseline date until December 31, 2012. The average follow-up was approximately 18 months and ranged from $<12$ to 48 months. Unadjusted all-cause mortality rate and the distribution of baseline potassium values for each disease state cohort and the control group were calculated for each $0.1 \mathrm{mEq} / \mathrm{L}$ change in potassium.

Cubic spline regression analysis was used to examine the relationship between baseline potassium values and mortality at 0.1 $\mathrm{mEq} / \mathrm{L}$ increments, adjusting for covariates of age, gender, HTN, CVD, AMI, CKD stages 3-5, HF, DM, RAASi, and diuretic prescriptions, along with multiple interactions (both age and gender with each comorbidity and RAASi, RAASi with CKD and with HF, and $\mathrm{HF}$ with $\mathrm{CKD}$; details in online suppl. Appendix). All statistical analyses were carried out using SAS/STAT software, version (9.2) of the SAS System for Windows.

\section{Results}

\section{Study Population}

Our sample comprised of 911,698 individuals with a mean age of $57.4 \pm 17.6 ; 64 \%$ were $<65$ years and $58 \%$ were female (Table 1; online suppl. Table S1). A total of $21 \%$ had $\operatorname{DM}(n=187,869), 11 \%$ had CKD $(n=97,384)$, and $6 \%$ had HF $(n=50,203)$, with $1 \%(n=9,821)$ having all 3 conditions. Diagnoses of HTN and CVD were present in $49 \%$ and $30 \%$, respectively. RAASi medications were prescribed in $30 \%$ of the study population and thiazide or loop diuretics in $19 \%$ and $6 \%$, respectively. At baseline, $27.6 \%$ had a potassium $<4.0$ and $5.7 \%$ had a value $\geq 5.0 \mathrm{mEq} / \mathrm{L}$.

The control group consisted of 338,297 individuals without known CVD, AMI, HTN, HF, CKD, or DM. Mild hypokalemia (potassium $3.5-<4.0 \mathrm{mEq} / \mathrm{L}$ ) was present in $26 \%$ of controls, while moderate-to-severe hypokalemia (potassium $<3.5 \mathrm{mEq} / \mathrm{L}$ ) occurred in $4.0 \%$. Hyperkalemia (with potassium values $\geq 5.0 \mathrm{mEq} / \mathrm{L}$ ) occurred in $4.3 \%$ of controls, $0.5 \%$ of whom had mod-severe hyperkalemia with potassium concentrations $\geq 5.5 \mathrm{mEq} / \mathrm{L}$. The total number of patients with potassium values $<4.0 \mathrm{mEq} / \mathrm{L}$ was numerically greater than those with potassium values $\geq 5.0 \mathrm{mEq} / \mathrm{L}$ in controls. Mild and moderate-to-severe hypokalemia occurred in $25.8 \%$ of those with HF, in $23.4 \%$ with $\mathrm{CKD}$, in $22.3 \%$ with $\mathrm{DM}$, and in $24.0 \%$ with all 3 conditions. Baseline potassium values $\geq 5.0 \mathrm{mEq} / \mathrm{L}$ varied across these disease states: $9.1 \%$ in HF, $11.5 \%$ with CKD stage $3-5,8.3 \%$ with DM, and $13.1 \%$ of those with all 3 conditions.

\section{Unadjusted Mortality by Baseline Potassium}

The relationship between serum potassium and allcause mortality in the control group and in HF, CKD, DM, and combined cohorts appears in Figure. 1. Eighteen-month mortality rates were $1.2 \%$ controls, $22.4 \%$ HF, $16.6 \% \mathrm{CKD}, 6.7 \% \mathrm{DM}$, and $29.7 \%$ with all 3 conditions. The lowest unadjusted mortality occurred with potassium values between 4.0 and $5.0 \mathrm{mEq} / \mathrm{L}$. Unadjusted mortality within this potassium range was 25 -fold higher in the combined cohort, 19-fold greater in HF, 14-fold greater in $\mathrm{CKD}$, and fivefold greater in $\mathrm{DM}$ compared to controls (online suppl. Table S2). All-cause mortality increased continuously with potassium values above or below the $4.0-5.0 \mathrm{mEq} / \mathrm{L}$ range in controls and all 4 predefined cohorts. Unadjusted death rates for hypokalemia and hyperkalemia in these cohorts were higher in $\mathrm{HF}$ when compared to those in CKD or DM.

All-cause mortality was highest $(25.7 \%$ at potassium values of $4.0-<5.0 \mathrm{mEq} / \mathrm{L}$ ) over 18 months in the combined cohort. We observed $45.5 \%$ mortality with moderate-to-severe hypokalemia and a $35.7 \%$ death rate with hyperkalemia in those with $\mathrm{HF}, \mathrm{CKD}$, and $\mathrm{DM}$, versus a death rate of $2.7 \%$ in controls at either extreme of the potassium values (Fig. 1d). In a comparison of older and younger comorbid patients, patients $\geq 65$ years with HF, $\mathrm{CKD}$, or DM had higher unadjusted mortality across all potassium levels versus subjects in the age range 50-64 years with any of the specified disease states (online suppl. Fig. S2, Table S3).

\section{Multivariate Predictors of High and Low \\ Serum Potassium}

The results of multivariate logistic regressions for predictors of mild and moderate-to-severe hypokalemia in individuals with baseline potassium $\leq 5.0 \mathrm{mEq} / \mathrm{L}$ and predictors of hyperkalemia in those with index potassium $\geq 4.0 \mathrm{mEq} / \mathrm{L}$ appear in Table 2 . CKD was predictive of both hyperkalemia and moderate-to-severe hypokalemia. Increasing age, male gender, DM, and RAASi prescriptions were all independent predictors of hyperkale- 
Table 1. Population characteristics, per distribution of baseline potassium values

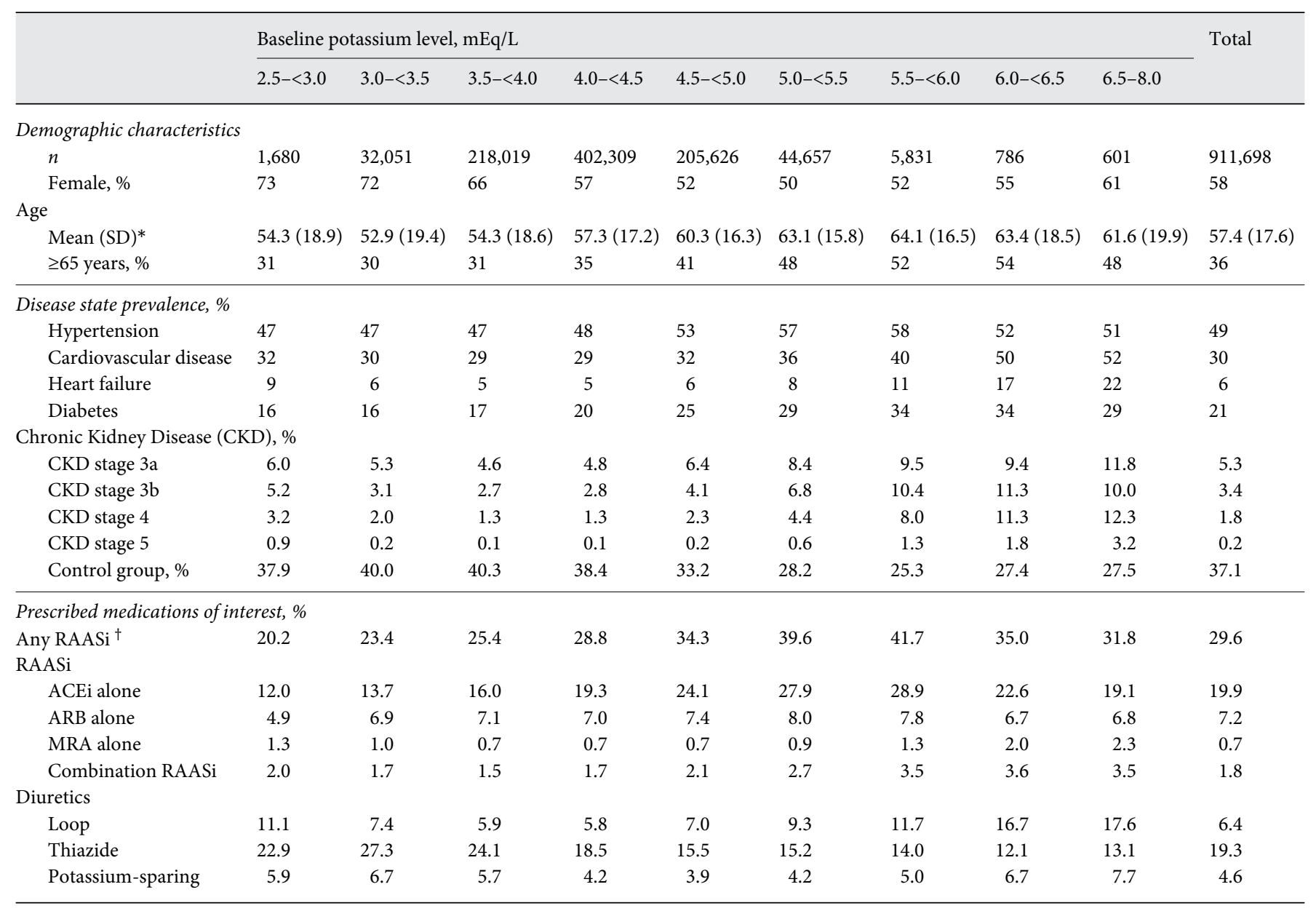

* Age was calculated on year of birth; those born in or before 1924 were assigned a birth year of 1924.

† Patients prescribed direct renin inhibitors are included only in the "Any RAASi" category.

$\mathrm{ACEi}$, angiotensin converting enzyme inhibitor; $\mathrm{ARB}$, angiotensin receptor blocker; $\mathrm{CKD}$, chronic kidney disease; MRA, mineralocorticoid receptor antagonist; RAASi, renin-angiotensin-aldosterone system inhibitors. Comorbidities are not mutually exclusive and may sum to more than $100 \%$. Data for potassium values of $8.1-10.0 \mathrm{mEq} / \mathrm{L}$ are not reported secondary to small sample size $(n=138)$.

mia, and independent negative predictors of both mild and moderate-to-severe hypokalemia; loop and thiazide diuretics showed the opposite pattern. CVD and HF independently predicted hypokalemia only. CKD was the best predictor of hyperkalemia, and odds ratios increased progressively with higher degrees of renal impairment. Thiazide diuretics doubled the predicted odds of hypokalemia and reduced the predicted odds of hyperkalemia by $36 \%$.

\section{Adjusted Mortality Association with Serum Potassium by Spline Analysis}

A U-shaped association was observed for serum potassium and adjusted mortality in the total population, in the control group, for $\mathrm{CKD}, \mathrm{HF}$, and $\mathrm{DM}$, and in the combined cohort, with mortality rates being the lowest at potassium levels between 4.0 and $<5.0 \mathrm{mEq} / \mathrm{L}$ (Fig. 2; online suppl. Fig. S3a-d, Table S4).

After controlling for covariates, spline analysis demonstrated that predicted mortality rates among subjects with HF, CKD, DM, and with all 3 conditions remained significantly higher at each potassium value relative to the control group (Fig. 2). With potassium values between 4.0 and $<5.0 \mathrm{mEq} / \mathrm{L}, 1.0 \%(1.0-1.1)$ of the control group was predicted to die during the follow-up versus $3.1 \%$ (2.93.3) of subjects with HF, 3.4\% (3.4-3.6) with CKD, $1.6 \%$ (1.6-1.7) with DM, and 7.5\% (6.9-8.1) with all 3 conditions (online suppl. Table S5). After adjusting for age, 


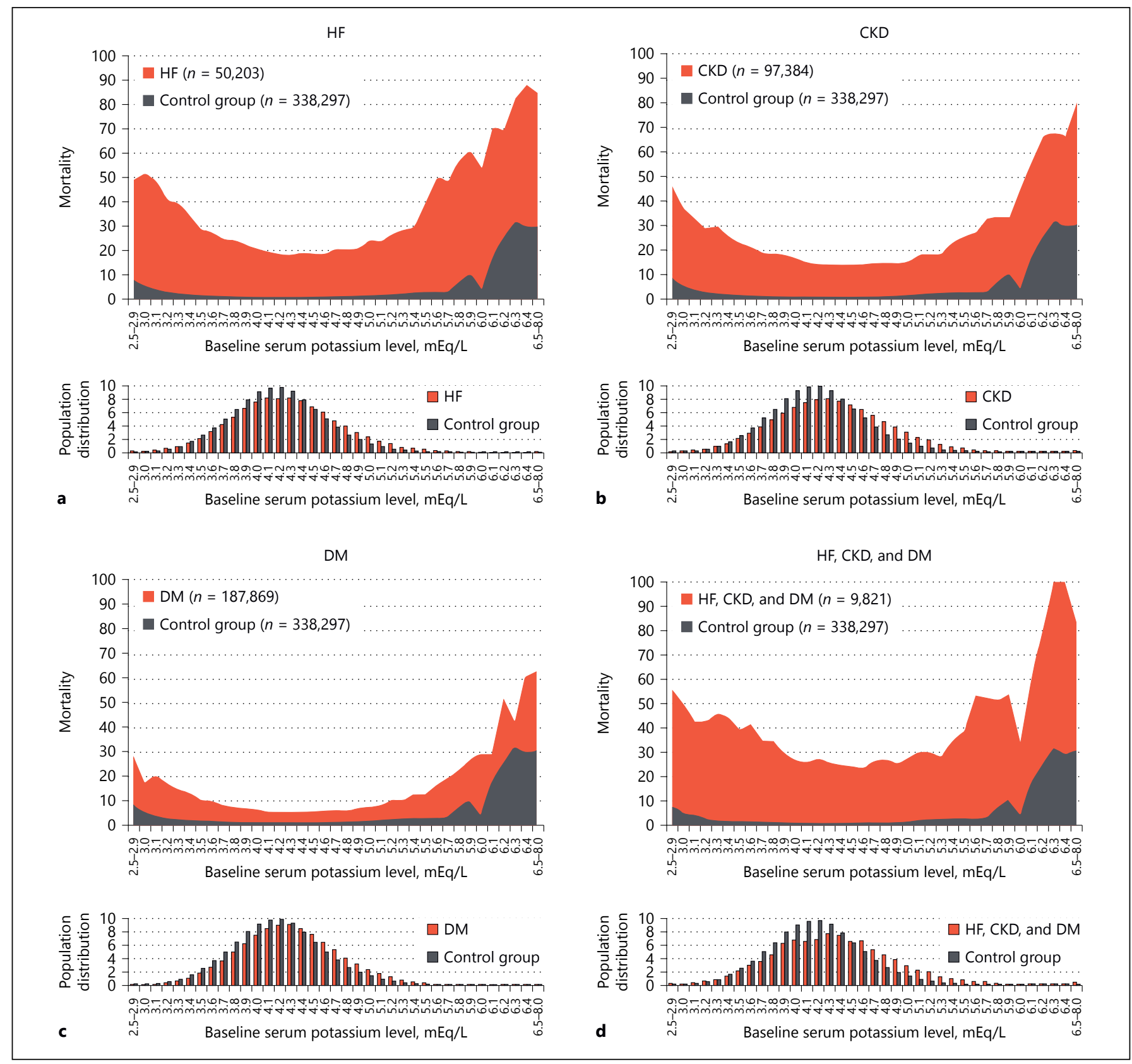

Fig. 1. Unadjusted mortality (percent) over 18 months and histogram (percent) of serum potassium values per $0.1 \mathrm{mEq} / \mathrm{L}$ increments $(2.5-8.0 \mathrm{mEq} / \mathrm{L})$ in a: $\mathrm{HF}, \mathbf{b}: \mathrm{CKD}, \mathbf{c}$ : DM, and $\mathbf{d}$ : combined cohort compared to controls. Data for patients with baseline serum

potassium of $8.1-10.0 \mathrm{mEq} / \mathrm{L}$ are not reported because the sample size was small $(n=138)$. CKD, chronic kidney disease; DM, diabetes mellitus, HF, heart failure.

gender, relevant comorbidities, and therapies, predicted mortality for HF, CKD, DM, and the combined cohort was greater for potassium values $\geq 5.0$ and $<4.0 \mathrm{mEq} / \mathrm{L}$ versus potassium values between 4.0 and $<5.0 \mathrm{mEq} / \mathrm{L}$. Cohorts with HF or CKD had similar predicted mortality risks across the distribution of potassium values (Fig. 2;

online suppl. Table S5). Among patients with HF, CKD, or DM, predicted mortality rates increased more markedly with mild dyskalemia in older subjects (age $\geq 65$ years) than in subjects in the age range 50-64, and were consistently higher across the entire distribution of serum potassium (online suppl. Fig. S4). 
Fig. 2. Spline analysis adjusted for covariates, showing serum potassium as a continuous variable with all-cause mortality over the distribution of potassium values (2.5-8.0 $\mathrm{mEq} / \mathrm{L})$ in $\mathrm{HF}, \mathrm{CKD}, \mathrm{DM}$, and combined cohort compared to controls. Data for patients with baseline serum potassium of $8.1-10.0 \mathrm{mEq} / \mathrm{L}$ are not reported because the sample size was small $(n=138)$. $\mathrm{CKD}$, chronic kidney disease; DM, diabetes mellitus, HF, heart failure.

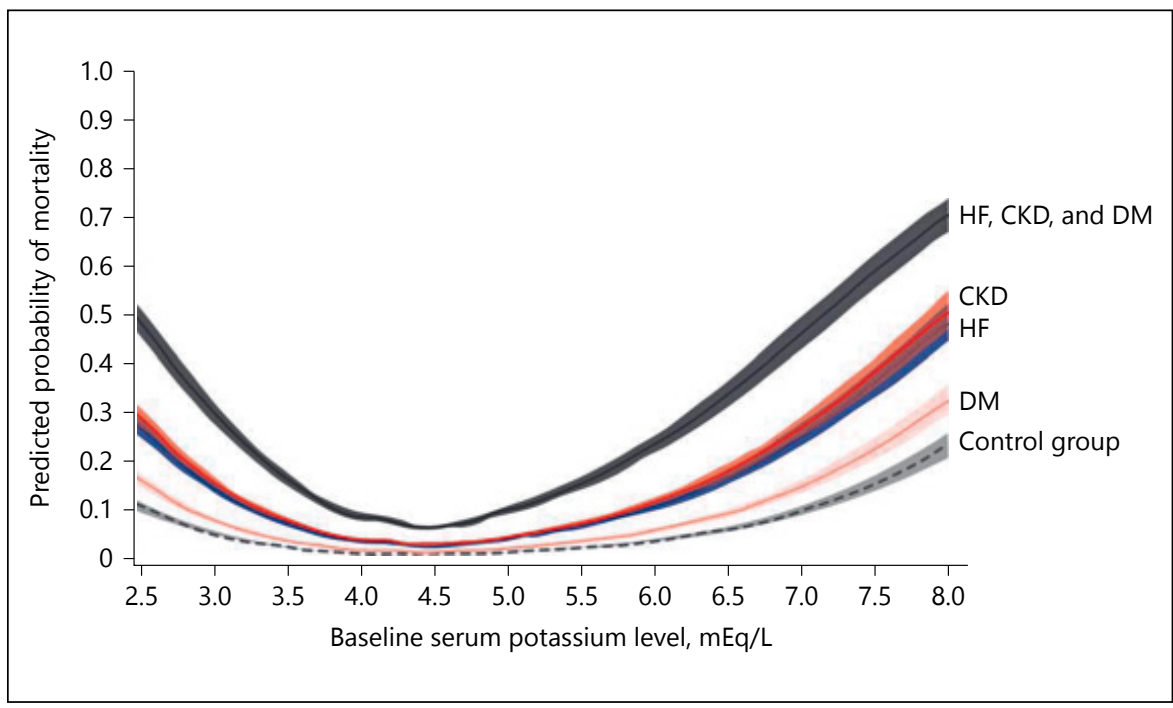

Table 2. Fully adjusted ORs for hypokalemia (serum potassium $<3.5 \mathrm{mEq} / \mathrm{L}$ and $<4.0 \mathrm{mEq} / \mathrm{L}$ ) and hyperkalemia (serum potassium $>5.0$ $\mathrm{mEq} / \mathrm{L}$ ) by covariate

\begin{tabular}{|c|c|c|c|}
\hline \multirow[t]{3}{*}{ Covariate, comparison } & \multicolumn{3}{|c|}{ Adjusted OR (95\% CI) } \\
\hline & \multicolumn{2}{|l|}{ hypokalemia } & \multirow{2}{*}{$\frac{\text { hyperkalemia }}{>5.0 \mathrm{mEq} / \mathrm{L}}$} \\
\hline & $<3.5 \mathrm{mEq} / \mathrm{L}$ & $<4.0 \mathrm{mEq} / \mathrm{L}$ & \\
\hline \multicolumn{4}{|l|}{ Age group } \\
\hline $45-64$ vs. $18-44$ & $0.53(0.51-0.54)$ & $0.57(0.56-0.58)$ & $1.41(1.36-1.47)$ \\
\hline $65-74$ vs. $18-44$ & $0.45(0.44-0.47)$ & $0.48(0.48-0.49)$ & $1.66(1.59-1.73)$ \\
\hline $75+$ vs. $18-44$ & $0.45(0.43-0.46)$ & $0.50(0.49-0.50)$ & $1.72(1.65-1.79)$ \\
\hline Gender, male vs. female & $0.59(0.57-0.60)$ & $0.65(0.64-0.66)$ & $1.14(1.11-1.16)$ \\
\hline HTN, 1 vs. 0 & $1.00(0.97-1.03)$ & $0.99(0.97-1.00)$ & $0.95(0.93-0.98)$ \\
\hline CVD, 1 vs. 0 & $1.19(1.16-1.22)$ & $1.15(1.14-1.17)$ & $1(0.98-1.03)$ \\
\hline AMI, 1 vs. 0 & $1.26(1.15-1.38)$ & $1.18(1.13-1.23)$ & $0.98(0.91-1.06)$ \\
\hline HF, 1 vs. 0 & $1.27(1.20-1.34)$ & $1.18(1.15-1.21)$ & $1.03(0.99-1.08)$ \\
\hline DM, 1 vs. 0 & $0.81(0.78-0.84)$ & $0.82(0.81-0.83)$ & $1.28(1.25-1.32)$ \\
\hline \multicolumn{4}{|l|}{ CKD stage } \\
\hline Stage 3 a vs. no-CKD/stages $1-2$ & $1.24(1.17-1.30)$ & $1.00(0.98-1.02)$ & $1.55(1.49-1.62)$ \\
\hline Stage $3 \mathrm{~b}$ vs. no-CKD/stages $1-2$ & $1.21(1.13-1.29)$ & $0.96(0.93-0.99)$ & $2.2(2.1-2.3)$ \\
\hline Stage 4 vs. no-CKD/stages $1-2$ & $1.51(1.39-1.64)$ & $0.99(0.95-1.03)$ & $2.97(2.82-3.13)$ \\
\hline Stage 5 vs. no-CKD/stages $1-2$ & $1.63(1.31-2.05)$ & $1.00(0.89-1.12)$ & $4.09(3.58-4.67)$ \\
\hline RAASi prescription, 1 vs. 0 & $0.61(0.59-0.63)$ & $0.68(0.67-0.69)$ & $1.34(1.31-1.38)$ \\
\hline Loop diuretic 1 vs. 0 & $1.44(1.38-1.51)$ & $1.21(1.19-1.24)$ & $0.95(0.91-0.99)$ \\
\hline Thiazide diuretic, 1 vs. 0 & $2.26(2.18-2.33)$ & $2.14(2.11-2.17)$ & $0.64(0.62-0.66)$ \\
\hline Potassium-sparing diuretic, 1 vs. 0 & $1.01(0.96-1.06)$ & $1.05(1.03-1.07)$ & $0.98(0.93-1.04)$ \\
\hline
\end{tabular}

The covariates: age group (18-44, 45-64, 65-74, and $\geq 75$ years); gender; HTN; CVD; AMI; CKD stage (no CKD/CKD stages 1-2, 3a, 3b, 4, or 5); HF; DM; RAASi (RAASi prescription in the 12 months pre-baseline); and loop, thiazide, and potassium-sparing diuretic prescriptions were used in logistic regression models as predictors of high potassium in patients with index potassium $\geq 4.0 \mathrm{mEq} / \mathrm{L}$, and as predictors of low potassium in patients with index potassium $\leq 5.0 \mathrm{mEq} / \mathrm{L}$. Age $<18$ years not shown.

AMI, acute myocardial infarction; CKD, chronic kidney disease; CVD, cardiovascular disease; DM, diabetes mellitus; HF, heart failure; HTN, hypertension; RAASi, renin-angiotensin-aldosterone system inhibitors. 


\section{Discussion}

Our analysis demonstrated that at baseline, one third of 911,698 patients receiving medical care from multiple healthcare providers across the United States had dyskalemia. We demonstrated a continuous U-shaped relationship between serum potassium and all-cause mortality in the entire population as well as in cohorts with HF, $\mathrm{CKD}, \mathrm{DM}$, and all 3 conditions, and in a control group without documented CVD, and aforementioned conditions. Dyskalemias including mild $(3.5-<4.0 \mathrm{mEq} / \mathrm{L})$ or moderate-to-severe $(<3.5 \mathrm{mEq} / \mathrm{L})$ hypokalemia and mild $(5.0-<5.5 \mathrm{mEq} / \mathrm{L})$ or moderate-to-severe hyperkalemia $(\geq 5.5 \mathrm{mEq} / \mathrm{L})$ were all associated with increased all-cause mortality over an average 18-month follow-up. Unadjusted 18-month mortality ranged from 34.8 to $55.6 \%$ with mild to severe hypokalemia and from 29.5 to $84.2 \%$ of those with mild-to-severe hyperkalemia in the cohort with HF, CKD, and DM. Fully adjusted all-cause mortality was higher across the entire distribution of serum potassium in those with HF, CKD, or DM and highest in the cohort with all 3 conditions versus controls.

After adjustment for multiple risk factors, the spline analysis demonstrated that patients with HF, CKD, DM, or all 3 conditions have significantly higher mortality rates than controls at all levels of serum potassium. Mortality was highest for all cohorts with mod-severe dyskalemia. Importantly, higher mortality occurred at potassium values that commonly engender little concern among clinicians, especially in those with HF or CKD. These findings are consistent with prior reports of U-shaped relationships between potassium and death in selected populations [10-13]. Individuals $\geq 65$ years of age had noticeably higher mortality rates for mild and moderate-to-severe hypokalemia and mild to moderate-tosevere hyperkalemia when compared to those aged 50 64 years.

Few studies have examined the relationship between dyskalemia and death in the absence of known CVD, HF, CKD, or DM. Unadjusted 18-month mortality was 1.2and 2.6-fold higher in individuals with potassium values $<4.5$ and 3.5 to $<4.0 \mathrm{mEq} / \mathrm{L}$, respectively in our control group, versus potassium levels of 4.0 to $<5.0 \mathrm{mEq} / \mathrm{L}$. Mortality was 1.9 -fold and 7.5-fold higher in controls with potassium values 5.0 to $<5.5$ and 5.5 to $8.0 \mathrm{mEq} / \mathrm{L}$, respectively, versus controls with potassium levels of 4.0 to $<5.0 \mathrm{mEq} / \mathrm{L}$.

Potassium levels $<4.0 \mathrm{mEq} / \mathrm{L}$ were more common than those $\geq 5.0 \mathrm{mEq} / \mathrm{L} ; 4.0 \%$ of the control group had moderate-to-severe hypokalemia, $26 \%$ had mild hypokalemia, and $4.3 \%$ had hyperkalemia. Thus, even in the absence of the aforementioned disease states, dyskalemia was associated with a higher risk of death.

The association of mild hypokalemia with greater allcause mortality in controls is noteworthy, since prior reports of higher mortality with mild hypokalemia (3.5$3.9 \mathrm{mEq} / \mathrm{L}$ ) have been limited to those with $\mathrm{HF}$ and to those with or without CKD. Our results showing a higher mortality risk for mod-severe hypokalemia differ from a recent report, which noted a $13 \%$ non-significant increase in death in subjects with potassium values $<3.5 \mathrm{mEq} / \mathrm{L}$, without known CVD [14].

A diagnosis of HF was reported in $5.1 \%$ of patients with potassium values $<4.0 \mathrm{mEq} / \mathrm{L}$, and in $6.1 \%$ with a potassium concentration $<3.5 \mathrm{mEq} / \mathrm{L}$. Potassium values $<4.0 \mathrm{mEq} / \mathrm{L}$ were associated with a 1.4 -fold increase in adjusted all-cause mortality versus potassium values 4.0 $<5.0 \mathrm{mEq} / \mathrm{L}$ in the HF cohort. Our findings are similar to prior analyses of HF patients, which reported greater allcause mortality and a trend to increased hospitalization in those with potassium values $<4.0 \mathrm{mEq} / \mathrm{L}[5,6]$. Fully adjusted odds ratios implicated HF, CVD, CKD, female gender, and loop or thiazide diuretics as risk factors for index potassium $<3.5 \mathrm{mEq} / \mathrm{L}$. Analysis of prescription data demonstrated a greater association for thiazide versus loop diuretics with mild and moderate-to-severe hypokalemia. Other diuretic-induced electrolyte abnormalities including hypomagnesemia and hyponatremia may contribute to the increase in mortality with hypokalemia [15].

Our results demonstrated incremental increases in allcause mortality in cohorts with HF, CKD, or DM or in combination, across potassium values $\geq 5.0 \mathrm{mEq} / \mathrm{L}$ following adjustment for covariates. Individuals with serum potassium of $\geq 5.0 \mathrm{mEq} / \mathrm{L}$ were more likely to be older, to be male, to have HF, CKD, or DM - especially CKD - and to receive RAASi therapy. Prescriptions for RAASi, primarily with angiotensin-converting enzyme inhibitors and angiotensin receptor blockers, were highest in those with a serum potassium $\geq 5.0 \mathrm{mEq} / \mathrm{L}$. Our analysis supports prior clinical observations, which have implicated age, renal impairment, and RAASi as risk factors for hyperkalemia $[16,17]$.

Drug therapies have long been implicated in dyskalemias. The higher death rates seen in those with HF, CKD, and DM with mild or moderate-to-severe hypokalemia could, in part, be attributable to diuretic induced hypokalemia with inadequate monitoring and/or potassium replacement. Dietary modification, potassium supplementation, and use of potassium-sparing diuretics may pre- 
vent hypokalemia, normalize serum potassium, and reduce risk of arrhythmia and mortality associated with hypokalemia. Few patients with hypokalemia were treated with potassium-sparing diuretics.

Hyperkalemia is an uncommon complication of RAASi in individuals without cardiac or renal disease and/or DM. Guideline-directed medical therapy with RAASi has oft been implicated as a major factor in the development of hyperkalemia in patients with HF, CKD, and/or DM. The development of hyperkalemia in patients with cardiac or renal disease prescribed RAASi poses a therapeutic dilemma, as those patients who are at highest risk for hyperkalemia are the same who may derive the greatest cardiovascular benefit from these medications $[18,19]$.

Management of chronic or recurrent hyperkalemia in patients with cardiac or renal disease has been problematic. The development of hyperkalemia in RAASi treated patients with $\mathrm{HF}, \mathrm{CKD}$, and/or DM frequently leads to down-titration or discontinuation of guideline-directed therapy with RAASi [16]. Diuretics alone or in combination with a low potassium diet have been the mainstay of treatment for drug-induced hyperkalemia. However, diuretics, especially in higher doses, may activate neurohormonal systems resulting in adverse effects in HF, CKD, and DM, thereby limiting chronic use [8]. Careful monitoring of renal function, electrolytes, and volume status is required when diuretics are prescribed for treatment of hyperkalemia in patients with cardiac and renal disease.

Newer potassium binders, which increase fecal excretion of potassium are now available and may be useful in the treatment of drug-induced hyperkalemia in patients with $\mathrm{HF}, \mathrm{CKD}$, and DM in the non-emergency setting. The FDA approved non-absorbed, potassium-binding polymer patiromer, which demonstrated effectiveness in the treatment of chronic or recurrent hyperkalemia in patients with CKD frequently complicated by HF or DM receiving RAASi [20-23]. An investigational agent, sodium zirconium cyclosilicate is also reported to be effective in treating hyperkalemia [23-25]. Although sodium polystyrene sulfonate has long been used in the semiacute setting, its chronic use is limited by gastrointestinal side effects, sodium load, and the risk of colonic necrosis $[18,26]$.

There are a number of important limitations to our study. Serum potassium was measured at only one point in time and analyzed in different laboratories. We were unable to adjust for intra-individual or interlaboratory variation in potassium measurement. Details on patients' race or ethnicity were not available from the data source.
Specific disease states and comorbidities were determined based on diagnostic codes, laboratory tests, and medical procedures; consequently, they may have been underidentified. Drug dosage, prescription fulfillment, medication compliance/adherence, and prescriptions for other drugs that may affect potassium were unavailable due to database limitations. The observation that mortality was increased in patients with $\mathrm{CKD}, \mathrm{HF}$, and $\mathrm{DM}$ relative to the comparison group at serum potassium levels between 4.0 and $<5.0 \mathrm{mEq} / \mathrm{L}$ may reflect confounding by indication, secondary to the presence of other disease or comorbidity. We were unable to adjust for other clinical and laboratory covariates, which were not systematically measured in the dataset, and this may contribute to residual confounding.

In summary, hypokalemia and hyperkalemia occurred commonly in a large, geographically diverse US population. Dyskalemia was associated with an increase in allcause mortality over an 18 month follow-up. Mortality risk was lowest with serum potassium values between 4.0 and $5 \mathrm{mEq} / \mathrm{L}$, both in those with and without CKD, HF, DM, and CVD. Associations between all-cause mortality and dyskalemia were consistently greater in $\mathrm{HF}, \mathrm{CKD}$ and $\mathrm{DM}$ disease. Hyperkalemia was more common in older patients, and had greater impact on all-cause mortality. Drug therapy was associated with dyskalemias, as prescriptions for thiazide/ loop diuretics were associated with mild and mod-severe hypokalemia; while RAASi prescriptions were linked to mild and mod-severe hyperkalemia.

Future clinical trials will be required to determine if aggressive management of hypokalemia and hyperkalemia may reduce mortality in patients with and without $\mathrm{HF}, \mathrm{CKD}$, DM, or CVD. In the interim, clinicians should monitor serum potassium with regularity and treat dyskalemias, especially in patients with CKD, HF, and DM receiving medications that may impact potassium levels.

\section{Acknowledgments}

This study was funded by Relypsa, Inc., a Vifor Pharma Group Company. Editorial support was provided by Julie Ann Obeid, an employee of Relypsa, Inc.; and by Narender K. Dhingra, of AlphaBioCom, LLC, which was funded by Relypsa, Inc.

\section{Disclosure Statement}

A.J.C. reports receiving research grants from Amgen, Hospira, NxStage, and ZS Pharma. He also reports receiving honoraria from Amgen and NxStage; and serving as a consultant/advisor to Amgen, NxStage, DaVita Clinical Research, Bayer, Relypsa, Inc., 
and Hospira. B.P. reports receiving personal fees for consulting with Sanofi, Relypsa, Merck, Bayer, AstraZeneca, Boehringer Ingelheim, Forest Laboratories, scPharmaceuticals, PharMain, Tricida, DaVinci Therapeutics, KBP Biosciences, Stealth Peptides, and AuraSense. He has stock options in scPharmaceuticals, PharMain, DaVinci Therapeutics, Tricida, KBP Biosciences, and AuraSense. He serves on data safety monitoring committees for and receives personal fees from Johnson \& Johnson, Novartis, and Tenax Pharmaceuticals. He serves on a clinical events committee and receives personal fees from Juventas Therapeutics. In addi- tion, he has a pending patent EFS ID 14916043, application number licensed to the University of Michigan School of Medicine. N.R. is the owner of Strategic Health Resources and reports serving as a consultant for Relypsa, Inc. S.F. reports serving as a consultant to Relypsa, Inc. and Strategic Health Resources. K.M. reports serving as a consultant for Relypsa, Inc., and Strategic Health Resources. D.W. is an employee of Relypsa, Inc. D.B. reports serving as a consultant for OPKO and Sanofi. He also reports serving as a consultant for, and having stock ownership with Amgen, Relypsa, Inc., and Tricida.

\section{References}

1 Palmer BF: Regulation of potassium homeostasis. Clin J Am Soc Nephrol 2015;10:10501060 .

2 Ahmed A, Husain A, Love TE, Gambassi G, Dell'Italia LJ, Francis GS, Gheorghiade M, Allman RM, Meleth S, Bourge RC: Heart failure, chronic diuretic use, and increase in mortality and hospitalization: an observational study using propensity score methods. Eur Heart J 2006;27:1431-1439.

3 Krijthe BP, Heeringa J, Kors JA, Hofman A, Franco OH, Witteman JC, Stricker BH: Serum potassium levels and the risk of atrial fibrillation: the Rotterdam Study. Int J Cardiol 2013;168:5411-5415.

4 Widimisky P: Hypokalemia and the heart. EJournal of Cardiology Practice 2008;7:9-12.

5 Ahmed A, Zannad F, Love TE, Tallaj J, Gheorghiade M, Ekundayo OJ, Pitt B: A propensity-matched study of the association of low serum potassium levels and mortality in chronic heart failure. Eur Heart J 2007;28: 1334-1343.

6 Bowling CB, Pitt B, Ahmed MI, Aban IB, Sanders PW, Mujib M, Campbell RC, Love TE, Aronow WS, Allman RM, Bakris GL, Ahmed A: Hypokalemia and outcomes in patients with chronic heart failure and chronic kidney disease: findings from propensity-matched studies. Circ Heart Fail 2010;3:253-260.

7 Wang $\mathrm{HH}$, Hung CC, Hwang DY, Kuo MC, Chiu YW, Chang JM, Tsai JC, Hwang SJ, Seifter JL, Chen HC: Hypokalemia, its contributing factors and renal outcomes in patients with chronic kidney disease. PLoS One 2013; 8:e67140.

8 Sarafidis PA, Georgianos PI, Bakris GL: Advances in treatment of hyperkalemia in chronic kidney disease. Expert Opin Pharmacother 2015;16:2205-2215.

9 Quinn J, Kramer N, McDermott D: Validation of the social security death index (SSDI): an important readily-available outcomes database for researchers. West J Emerg Med 2008;9:6-8.
10 Kovesdy CP, Regidor DL, Mehrotra R, Jing J, McAllister CJ, Greenland S, Kopple JD, Kalantar-Zadeh K: Serum and dialysate potassium concentrations and survival in hemodialysis patients. Clin J Am Soc Nephrol 2007;2: 999-1007.

11 Torlen K, Kalantar-Zadeh K, Molnar MZ, Vashistha T, Mehrotra R: Serum potassium and cause-specific mortality in a large peritoneal dialysis cohort. Clin J Am Soc Nephrol 2012;7:1272-1284.

12 Nakhoul GN, Huang H, Arrigain S, Jolly SE, Schold JD, Nally JV Jr, Navaneethan SD: Serum potassium, end-stage renal disease and mortality in chronic kidney disease. Am J Nephrol 2015;41:456-463.

13 Hessels L, Hoekstra M, Mijzen LJ, Vogelzang M, Dieperink W, Lansink AO, Nijsten MW: The relationship between serum potassium, potassium variability and in-hospital mortality in critically ill patients and a before-after analysis on the impact of computer-assisted potassium control. Crit Care 2015;19:4.

14 Hughes-Austin JM, Rifkin DE, Beben T, Katz R, Sarnak MJ, Deo R, Hoofnagle AN, Homma S, Siscovick DS, Sotoodehnia N, Psaty BM, de Boer IH, Kestenbaum B, Shlipak MG, Ix JH: The relation of serum potassium concentration with cardiovascular events and mortality in community-living individuals. Clin J Am Soc Nephrol 2017;12:245-252.

15 Sica DA: Diuretic-related side effects: development and treatment. J Clin Hypertens (Greenwich) 2004;6:532-540.

16 Epstein M: Hyperkalemia constitutes a constraint for implementing renin-angiotensinaldosterone inhibition: the widening gap between mandated treatment guidelines and the real-world clinical arena. Kidney Int Suppl 2016;6:20-28.

17 Ferrario CM: New physiological concepts of the renin-angiotensin system from the investigation of precursors and products of angiotensin I metabolism. Hypertension 2010;55: 445-452.
18 Palmer BF: Managing hyperkalemia caused by inhibitors of the renin-angiotensin-aldosterone system. N Engl J Med 2004;351:585592.

19 Sarwar CM, Papadimitriou L, Pitt B, Piña I, Zannad F, Anker SD, Gheorghiade M, Butler J: Hyperkalemia in heart failure. J Am Coll Cardiol 2016;68:1575-1589.

20 VELTASSA $^{\mathrm{TM}}$ (Patiromer) for Oral Suspension [Full Prescribing Information]. Redwood City, Relypsa, Inc., 2016.

21 Weir MR, Bakris GL, Bushinsky DA, Mayo MR, Garza D, Stasiv Y, Wittes J, ChristSchmidt H, Berman L, Pitt B; OPAL-HK Investigators: Patiromer in patients with kidney disease and hyperkalemia receiving RAAS inhibitors. N Engl J Med 2015;372:211-221.

22 Ingelfinger JR. A new era for the treatment of hyperkalemia? N Engl J Med 2015;372:275277.

23 Bakris GL, Pitt B, Weir MR, Freeman MW, Mayo MR, Garza D, Stasiv Y, Zawadzki R, Berman L, Bushinsky DA; AMETHYST-DN Investigators: Effect of patiromer on serum potassium level in patients with hyperkalemia and diabetic kidney disease: the AMETHYSTDN randomized clinical trial. JAMA 2015; 314:151-161.

24 Packham DK, Rasmussen HS, Lavin PT, ElShahawy MA, Roger SD, Block G, Qunibi W, Pergola P, Singh B: Sodium zirconium cyclosilicate in hyperkalemia. N Engl J Med 2015; 372:222-231.

25 Kosiborod M, Rasmussen HS, Lavin P, Qunibi WY, Spinowitz B, Packham D, Roger SD, Yang A, Lerma E, Singh B: Effect of sodium zirconium cyclosilicate on potassium lowering for 28 days among outpatients with hyperkalemia: the HARMONIZE randomized clinical trial. JAMA 2014;312:22232233.

26 Leaf DE, Cheng XS, Sanders JL, Mendu M, Schiff GD, Mount DB, Bazari H: An electronic alert to decrease Kayexalate ordering. Ren Fail 2016;38:1752-1754. 